\title{
Focusing on goals
}

December is AIDS month. Forty years after the syndrome we now know as AIDS was first seen in the USA, the disease is still with us. It should not be.

An estimated 37.7 million people globally were living with HIV in 2020; 1.5 million people were newly infected with HIV; and 680000 people died from AIDS-related illnesses. ${ }^{[1]}$ Sub-Saharan Africa carries $67 \%$ of this burden, with 25.7 million people living with the disease. More than $60 \%$ of new HIV infections globally occur in the African region, and there were 470000 AIDS-related deaths in 2020. ${ }^{[2]}$

The World Health Organization (WHO) is committed to ending the AIDS epidemic as a public health threat by $2030 .{ }^{[3]}$ The targets of this strategy, in partnership with multiple international bodies, are the familiar 95-95-95 goals - 95\% diagnosed among all people living with HIV, 95\% on antiretroviral treatment among those diagnosed, and, among those on treatment, 95\% virally suppressed. Although there have been impressive results, the gap between achievements to date and the 2030 goal is too wide. The Joint United Nations Programme on HIV/AIDS (UNAIDS) has put in place a 'fast-track' plan to accelerate achieving these goals. ${ }^{[4]}$ In essence, this encourages countries to alter their approach to managing HIV and AIDS, with an emphasis on differentiated service delivery, adapting where necessary for maximum efficacy and efficiency. The focus is on modifying the delivery of HIV services in order to do this - changing paradigms of testing, access to treatment, monitoring of treatment and allocation of resources. There is a strong push to utilise primary health facilities and community-based services to reach as many people as possible and retain them in the care cascade. This approach is rooted in the second dimension of universal health coverage - addressing how these services can be delivered. The best methods deliver the continuum of HIV services to different populations, in different locations, in order to achieve the maximum impact and ensure quality. ${ }^{[3]} \mathrm{A}$ range of health providers should be skilled in all elements of HIV care, providing testing, treatment and follow-up, and monitoring. This is particularly important when reaching key populations, such as men who have sex with men and partners of those living with the disease.

And where are we in southern Africa? A target of a 75\% reduction in new cases by 2020 was set in 2010 . Sadly, there has only been an overall $42 \%$ reduction, a considerable gap. South Africa (SA) has only achieved a $45 \%$ reduction, far below Eswatini and Malawi (both at $64 \%)$. While SA is doing well in terms of the percentage of people who know their status (92\%) and relatively well in terms of the percentage of those people who are on treatment (75\%), we fall quite far down in regional terms when it comes to the percentage of people living with HIV who are virally suppressed. Here the figure is only $61 \%$, a long way behind Botswana, Namibia, Eswatini, Malawi, Lesotho, Zambia and even Zimbabwe, who range between $89 \%$ and $98 \%$ (Southern African Development Community 2020 HIV and AIDS Epidemic Report, personal communication).

Given that $75 \%$ of those who know their status are on treatment, the lack of viral suppression is worrying, suggesting that adherence and retention is a problem. It is here that the global strategies are most important - shifting tasks among health providers, differentiating service delivery, and focusing on ensuring not only that patients have easy access to antiretrovirals, but that they are provided in a way that encourages adherence and retention in care.

If we are to reach the target of eliminating AIDS by 2030, we need to be totally inclusive in our approach to managing our patients. What matters is the patient and their community. This is where our focus should be.

\section{Bridget Farham}

Editor

ugqirha@iafrica.com

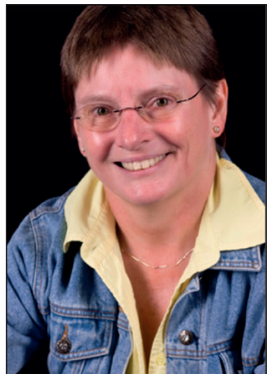

1. Joint United Nations Programme on HIV/AIDS (UNAIDS). Global HIV and AIDS statistics. https:// www.unaids.org/en/resources/fact-sheet (accessed 17 November 2021).

2. World Health Organization. HIV/AIDS statistics. https://www.afro.who.int/health-topics/hivaid (accessed 17 November 2021).

3. World Health Organization. Global health sector strategy on HIV: 2016 - 2021 - towards endin AIDS. https://apps.who.int/iris/bitstream/handle/10665/246178/WHO-HIV-2016.05-eng.pdf (accessed 17 November 2021)

4. Joint United Nations Programme on HIV/AIDS (UNAIDS). Fast-track: accelerating action to en to the AIDS epidemic by 2030. https://www.unaids.org/en/resources/documents/2014/JC2686 WAD2014report (accessed 17 November 2021).

S Afr Med J 2021;111(12):1159. https://doi.org/10.7196/SAMJ.2021.v111i12.16283 